\title{
Effects of aerobic exercise therapy and cognitive behavioural therapy on functioning and quality of life in amyotrophic lateral sclerosis: protocol of the FACTS-2-ALS trial
}

Annerieke C van Groenestijn ${ }^{1}$, Ingrid GL van de Port ${ }^{1 *}$, Carin D Schröder ${ }^{1}$, Marcel WM Post ${ }^{1,2}$, Hepke F Grupstra ${ }^{4}$, Esther T Kruitwagen ${ }^{1}$, Harmen van der Linde ${ }^{5}$, Reinout $\mathrm{O}$ van Vliet ${ }^{3}$, Margreet GH van de Weerd ${ }^{1}$, Leonard $\mathrm{H}$ van den Berg ${ }^{1}$ and Eline Lindeman ${ }^{1}$

\begin{abstract}
Background: Amyotrophic lateral sclerosis (ALS) is a fatal progressive neurodegenerative disorder affecting motor neurons in the spinal cord, brainstem and motor cortex, leading to muscle weakness. Muscle weakness may result in the avoidance of physical activity, which exacerbates disuse weakness and cardiovascular deconditioning. The impact of the grave prognosis may result in depressive symptoms and hopelessness. Since there is no cure for ALS, optimal treatment is based on symptom management and preservation of quality of life (QoL), provided in a multidisciplinary setting. Two distinctly different therapeutic interventions may be effective to improve or preserve daily functioning and QoL at the highest achievable level: aerobic exercise therapy (AET) to maintain or enhance functional capacity and cognitive behavioural therapy (CBT) to improve coping style and cognitions in patients with ALS. However, evidence to support either approach is still insufficient, and the underlying mechanisms of the approaches remain poorly understood. The primary aim of the FACTS-2-ALS trial is to study the effects of AET and CBT, in addition to usual care, compared to usual care alone, on functioning and QoL in patients with ALS.
\end{abstract}

Methods / Design: A multicentre, single-blinded, randomized controlled trial with a postponed information model will be conducted. A sample of 120 patients with ALS (1 month post diagnosis) will be recruited from 3 university hospitals and 1 rehabilitation centre. Patients will be randomized to one of three groups i.e. (1) AET + usual care, (2) CBT + usual care, (3) Usual care. AET consists of a 16-week aerobic exercise programme, on 3 days a week. CBT consists of individual psychological support of patients in 5 to 10 sessions over a 16-week period. QoL, functioning and secondary outcome measures will be assessed at baseline, immediately post intervention and at 3- and 6months follow-up.

Discussion: The FACTS-2-ALS study is the first theory-based randomized controlled trial to evaluate the effects, and the maintenance of effects, of AET and CBT on functioning and QoL in patients with ALS. The results of this study are expected to generate new evidence for the effect of multidisciplinary care of persons with ALS.

Trial registration: Dutch Trial Register NTR1616.

\footnotetext{
* Correspondence: i.v.d.port@dehoogstraat.nl

${ }^{1}$ Rudolf Magnus Institute of Neuroscience and Centre of Excellence for Rehabilitation Medicine, University Medical Centre Utrecht and Rehabilitation Centre De Hoogstraat, the Netherlands

Full list of author information is available at the end of the article
} 


\section{Background}

Amyotrophic lateral sclerosis (ALS) is a fatal progressive neurodegenerative disorder affecting motor neurons in the spinal cord, brainstem, and motor cortex. Patients are afflicted by progressive wasting and weakness of limb, bulbar, and respiratory muscles, and die on average within 3 years after symptom onset, usually because of respiratory failure [1]. In the absence of an established biological marker, the diagnosis of ALS is primarily clinical, based on El Escorial criteria [2]. The median age of onset of ALS is 55 years [1]. The incidence of ALS is between 1.5 and 2.5 per 100,000 person-years of followup in industrialized countries [3], and life-time risk of ALS is estimated to be between $1 / 600$ and $1 / 2000$ [1,4], which makes it the most common motor neuron disease.

ALS is familial in $5 \%$ of cases, with a Mendelian pattern of inheritance. The clinical phenotype of familial ALS (FALS) is similar to that of the sporadic form of the disease. At least 13 genes of major effect have been associated with FALS, accounting for $30 \%$ of familial cases. Sporadic ALS is considered to be a complex genetic disease, in which genetic and environmental factors combine to increase the disease risk [5].

Symptoms presented in the early stages of ALS may vary, and typically result from the combination of lower motor neuron loss (atrophy, fasciculations, weakness) and upper neuron motor loss (spasticity, pathological reflexes) $[1,6]$. Most patients show either progressive asymmetric focal weakness of the upper and lower extremities, e.g. poor handgrip or stumbling $(80 \%)$, or bulbar symptoms, e.g. dysarthria, dysphagia $(20 \%)[1,6]$. Muscle weakness caused by ALS may also result from the avoidance of physical activity, which may result in cardiovascular deconditioning and disuse weakness, superimposed on the weakness caused by the ALS itself [7]. If the reduced level of activity persists, further deconditioning can develop, and muscle and joint tightness may lead to contractures and pain. All these aspects hamper daily activities [7]. Besides physical limitations, cognitive function deficits in a frontotemporal pattern may be apparent, including impaired frontal executive abilities in up to $50 \%$ of patients [8]. The grave prognosis of ALS can have a severe psychological impact on patients and their social environment. The majority of patients report not only physical but also existential problems [9]. Depressive symptoms [10] and hopelessness $[10,11]$ are more common in patients with ALS than in the general population.

To date, no curative treatment is available. Because ALS symptoms are multifactorial, usual care for patients with ALS in the Netherlands consists of multidisciplinary care by specialized ALS care teams, coordinated by a rehabilitation physician.
The aim of our study is to improve or preserve functioning and quality of life (QoL) in patients with ALS. "Functioning" in this study refers to the domains of "activities" and "participation", as defined in the International Classification of Functioning, Disability and Health (ICF) [12], including functional status, physical activity, participation restrictions, and autonomy in participation. "QoL" is a construct described as "an individual integration of physical aspects such as symptoms of illness or wellness, psychological aspects such as emotional responses and beliefs, and social aspects such as interpersonal relationships and social support" [13]. Two distinctly different therapeutic interventions may be hypothesized to support the QoL of patients with ALS and preserve functioning at the highest achievable level, viz. aerobic exercise therapy (AET) to maintain or increase functional capacity, and cognitive behavioural therapy (CBT) to improve coping style and cognitions in patients with ALS. However, evidence to support the effectiveness of either approach is still limited.

The practice of prescribing exercise programmes for people with ALS is controversial, as some experts have discouraged exercise programmes for patients with neuromuscular disease for fear of "overuse weakness" [14-16]. Epidemiologic data showing a higher incidence of ALS in people who had engaged in intense physical activity during work or leisure before the onset of the disease has led to reluctance to prescribe exercise for people with ALS [17].

On the other hand, in a recent Cochrane review on muscle strength training and aerobic exercise training for patients with neuromuscular diseases (NMD), Voet [18] concluded that moderate-intensity strength training appeared not to be harmful to patients with myotonic dystrophy (MD) and facioscapulohumeral muscular dystrophy (FSHD), though there was insufficient evidence to establish its benefits. In a systematic review, Cup [19] summarized and critically appraised the available evidence on exercise therapy and other types of physical therapy for patients with NMD, including ALS. Again, exercise training did not seem to harm these patients, but insufficient evidence was reported for the effects of strengthening exercises, aerobic exercises or lifestyle modifications in the domains of body functions, activities, or participation for patients with ALS. The Cochrane review by Dal Bello [7] included only two small RCTs on exercise training for patients with ALS, and the small sample sizes made it impossible to draw conclusions about the effect of strengthening exercises for people with ALS. The authors recommended conducting larger, controlled studies to elucidate the effect of strengthening or aerobic exercise in people with ALS. In addition, better controlled studies are needed to 
determine which exercise protocols are most beneficial or cause undue risks, and whether there is a sub-set of people with ALS who respond more favourably to exercise, both physically and psychologically [7].

In addition to AET, it might be beneficial to alter coping styles and illness cognitions in patients with ALS by means of cognitive behavioural therapy (CBT), in order to improve or preserve functioning and QoL. Coping style and illness cognitions seem to be much more important than disease severity for the QoL of patients with ALS [20-22]. A passive coping style is a strong and consistent determinant of decreased QoL in patients with ALS and their primary caregivers [22]. In a crosssectional study by Matuz [21], multiple regression analysis revealed that $56 \%$ of the variance of QoL was explained by social support, coping strategies, and cognitive appraisal. Another psychological factor that has been shown to explain variance in activity limitations in patients with ALS is emotional wellbeing [23]. A psychological intervention like CBT, aiming to optimize coping style and cognitions, has been proven to be effective in terms of QoL in patients with multiple sclerosis suffering from depression [24], and in terms of physical functioning in patients with chronic fatigue syndrome $[25,26]$ and severely fatigued disease-free cancer patients [27]. This may suggest that CBT can also be beneficial to patients with ALS. However, no evidence for the effectiveness of CBT in patients with ALS is currently available.

The primary objectives of the FACTS-2-ALS trial are:

- Studying the effects of AET in addition to usual care, compared to usual care alone, on functioning and QoL in patients with ALS.

- Studying the effects of CBT in addition to usual care, compared to usual care alone, on functioning and $\mathrm{QoL}$ in patients with ALS.

Secondary objectives of the FACTS-2-ALS trial are:

- Studying the effects of AET in addition to usual care in patients with ALS on the ICF domains of body functions, and environmental and personal factors.

- Studying the effects of CBT in addition to usual care in patients with ALS on the ICF domains of body functions, and environmental and personal factors.

\section{Methods/Design Study design}

A multicentre, single-blinded, randomized controlled trial (RCT) with 6 months follow-up will be conducted to evaluate the effects of aerobic exercise therapy (AET) in addition to usual care, or cognitive behavioural therapy (CBT) in addition to usual care, compared to usual care alone, in patients with ALS (figure 1). Participants will first be invited to participate in a longitudinal study including four repeated measurements (first permission phase): at entry (T0), at 4 months (T1), at 7 months (T2), and at 10 months (T3). After randomization to one of the intervention groups, the participants will be informed about the intervention they have been assigned to, and will be asked to participate in this intervention (second permission phase). The interventions will take place between $\mathrm{T} 0$ and $\mathrm{T} 1$, making $\mathrm{T} 2$ a short-term and T3 a long-term follow-up for those who participate in an intervention. The patients in the usual care group will not be asked additional permission. This postponed information model [28] allows us to inform participants about part of the study only, while the rest of the information will be given to them at the end of the study.

The study protocol has been approved by the Medical Ethics Committee of the University Medical Centre Utrecht (UMCU), and all participating centres have agreed to participate. Informed consent according to the declaration of Helsinki will be obtained from all participants (in both the first and second permission phases).

The FACTS-2-ALS trial is part of the FACTS-2-NMD project, along with the FACTS-2-PPS [29] and FACTS2-FSHD [30] trials. An additional study will analyse qualitative data on patients' and partners' expectations of, and experiences with, the interventions, using a responsive methodology for all 3 trials [31].

\section{Study population}

The aim is to include 120 patients with ALS, one month post diagnosis, who meet the inclusion and exclusion criteria (table 1). All patients will be recruited from outpatient clinics of the UMCU, the Academic Medical Centre (AMC) in Amsterdam, the University Medical Centre Nijmegen (UMCN) or het Roessingh Rehabilitation Centre in Enschede. The neurologists will refer eligible patients to a rehabilitation physician, who will check the inclusion and exclusion criteria. If a patient meets all the criteria, the rehabilitation physician will inform them about the four measurements, and will ask permission for them to be contacted by the primary investigator. The primary investigator will invite the patients by telephone to take part in the four measurements (first permission phase), and (for those patients randomized to one of the interventions) to participate in the relevant intervention (second permission phase).

\section{Randomization and blinding}

Eligible participants will be randomized to one of three groups: (1) AET + usual care, (2) CBT + usual care, or (3) usual care. Patients who exercise for two or more hours a week will be excluded from the AET intervention, due to the small expected treatment effect, but can be randomized to the $\mathrm{CBT}$ or control group. In addition, since CBT is not expected to be necessary for patients who show no anxious or depressive symptoms, 


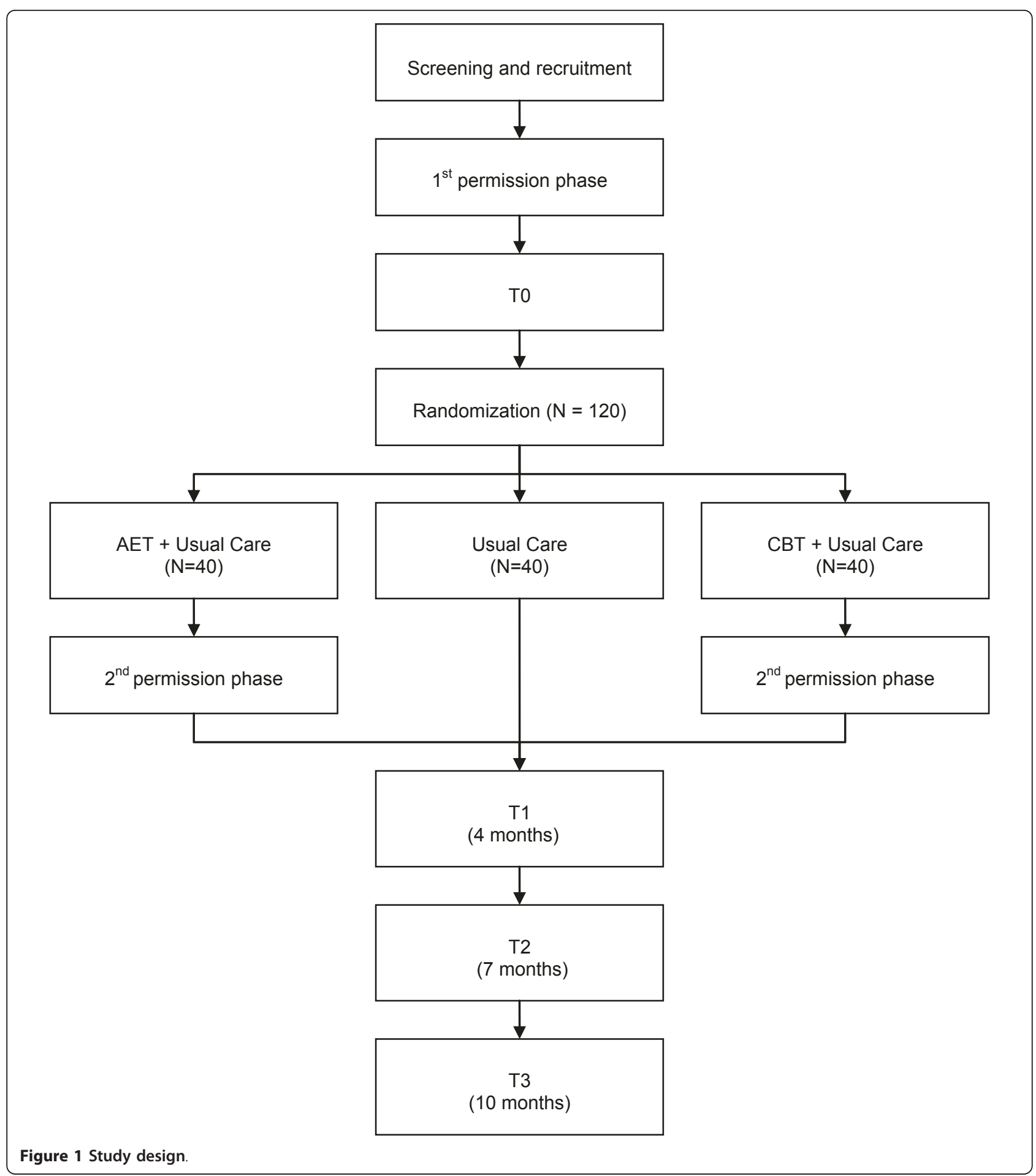

patients who score less than 8 on the Hospital Anxiety and Depression Scale (HADS) [32] will be excluded from the CBT intervention, but can be randomized to the AET or control group. All participating patients will be registered and randomized by one independent person. Randomization of the three trial arms will be implemented by the "minimization" method [33], a dynamic randomization algorithm that ensures even distribution of patients across strata, even if there are relatively small groups of patients to be divided over multiple strata. In this study, patients will be stratified by (a) study site (Amsterdam, Nijmegen, Enschede, 


\section{Table 1 Inclusion and exclusion criteria}

\begin{tabular}{l}
\hline Inclusion criteria \\
\hline (1) age between 18 and 70 years \\
\hline (2) life-expectancy longer than one year \\
\hline (3) forced vital capacity at least $80 \%$ \\
(4) diagnosis of "Probable" of "definite" ALS according to the "revised El \\
Escorial WFN criteria" [2] \\
(5) at least 1 month after the diagnosis of ALS \\
(6) being in the rehabilitation phase; diagnostic phase is completed \\
(7) walking ability with or without a ankle-foot orthosis or stick, and \\
cycling ability on a cycle ergometer, so that the intervention can be \\
expected to be completed \\
Exclusion criteria \\
(1) cognitive impairment \\
\hline (2) insufficient mastery of the Dutch language \\
(3) disabling co-morbidity interfering with the intervention programmes \\
or influencing outcome parameters (including severe cardiopulmonary \\
disease, like chest pain, arrhythmia, pacemaker, cardiac surgery, severe \\
dyspnoea on effort or emphysema, epileptic seizures, poorly regulated \\
diabetes mellitus or hypertension \\
(4) psychological disorder preventing the intervention from being \\
completed
\end{tabular}

Utrecht); (b) age; (c) the use of lithium; (d) type of ALS (spinal / bulbar); and (e) gender. A biphasic randomization of patients will be used, based on a postponed information model [28]. All outcomes will be assessed by blinded and independent research assistants. At the beginning of each assessment, patients will be instructed not to reveal their intervention to the blinded research assistant.

\section{Interventions \\ Usual Care}

The patients in the control and intervention groups will receive usual care. In the Netherlands, patients with ALS receive multidisciplinary care from specialized ALS care teams consisting of a rehabilitation physician, a physical therapist, an occupational therapist, a speech pathologist, a dietician, and a social worker. The ALS team works according to the Dutch protocol for rehabilitative management in ALS [34]. Rehabilitation medicine plays an important role in the symptomatic and palliative treatment of patients with ALS. The purpose of the rehabilitation treatment is to reduce the impact of the disabilities, so that patients can fulfil the roles they may wish and need to. Maintaining QoL is the primary objective. Rehabilitation treatment includes instructions on safety in mobility, use of aids and appliances, orthoses, and psychosocial guidance. Patients with ALS often receive physical therapy periodically, whenever new mobility problems arise. This therapy usually comprises compensatory strategies. In later stages of the disease, patients mostly receive physical therapy at home by a physical therapist, including passive mobility exercises, with practical goals to help them function safely at home. If necessary, the rehabilitation physician refers a patient to a social worker for social support. If there is a particular presenting complaint regarding mood disturbances, the patient can be referred to a psychologist.

Patients included in our trial will not be restricted in terms of their treatment options in the context of usual care; co-interventions such as exercise therapy and/or psychotherapy will be monitored throughout the study by means of diaries.

\section{Aerobic Exercise Therapy (AET)}

The AET will consist of a 16-week aerobic exercise programme, on 3 days a week, twice a week at home (1) and once a week in an individually guided group session at a hospital (2). The therapy will be supervised by specially trained physiotherapists.

(1) The training programme at home will consist of individually tailored aerobic exercises on a cycle ergometer and a stepboard. Patients will receive a Polar RS400 heart rate watch with chest strap, a Kettler X7 cycle ergometer, a Kettler stepboard, a log book with training instructions and a training schedule to be used at home for the duration of the intervention. During each training session, their heart rate will be recorded continuously by a (Polar RS400) heart rate monitor. Furthermore, the patients will use the log book to document the number and duration of training sessions, the training intensity, perceived exertion on the Borg Rated Perceived Exertion (RPE) scale [35], and possible complaints experienced after the training session. Each session will consist of a cycle ergometer training, followed by a stepboard training. The duration of the cycle ergometer training sessions will be gradually increased from 15 to 30 minutes per session in the first four weeks, after which the duration of training will be maintained at 30 minutes. The duration of the stepboard training session will be gradually increased from 3 minutes (weeks 1 - 5) to 4 minutes (weeks 6 - 10) to 5 minutes (weeks 11 - 16) per session. This training structure is based on expert opinion, since there have been no previous studies on aerobic exercise training in persons with ALS. Training intensity will be gradually increased from $50 \%$ of the Heart Rate Reserve (HRR) to 75\% HRR, in accordance with the American College of Sports Medicine guidelines [36] for aerobic training in healthy adults and persons with chronic diseases and disabilities [37]. HRR is the difference between the predicted maximum heart rate (220 - age) and the measured resting heart rate. The HRR is equivalent to the difference between maximal and resting maximal oxygen consumption (VO2max). Each participant will be instructed how to adjust the physical intensity to their prescribed individual heart rate. 
Warming-up and cooling-down will consist of 5 minutes unloaded cycling.

(2) The supervised group training sessions at the hospital will consist of workstations, including individually tailored aerobic exercises and muscle strengthening exercises. Sessions will be divided into a 5-minute warming-up period, 30 minutes of aerobic exercises (cycle ergometer, stepboard, and treadmill) and 20 minutes of muscle strengthening exercises (quadriceps, biceps, and triceps) and a 5-minute cooling-down period.

Aerobic exercise workstations: training intensity will be gradually increased from $50 \%$ of the HRR to $75 \%$ HRR. Exercise duration per workstation will remain constant, i.e. cycle ergometer 15 minutes; treadmill 10 minutes; stepboard 3 to 5 minutes. After each aerobic workstation, duration and heart rate (as determined by the heart rate monitor) as well as the perceived exertion on the Borg RPE scale, will be recorded in the log book by the physical therapist.

Muscle strengthening exercises at workstations: the maximum strength (1-RM) of different muscle groups in arms and legs will be determined individually using a submaximal test. Since muscle strength is expected to deteriorate over time in patients with ALS, the physical therapist will determine the maximum strength before the start of the programme, and again after 5 and 10 weeks of training, to ensure that the patients exercise at the correct training intensity. In the first 5 weeks, training intensity will be $40 \%$ of $1-\mathrm{RM}$, with each exercise session consisting of 3 series of 10 repetitions each, with 1 minute of rest between series. Training intensity will be increased to $50 \%$ of 1 - RM in week 6 , and in week 11 the number of repetitions will be increased to 15. After each muscle strengthening exercise, the therapist will record the actual training intensity achieved and the number of series and repetitions in the logbook, as well as the perceived exertion on the Borg RPE scale. This progressive, moderate intensity is in accordance with the intensity of the exercise programme for persons with ALS developed by Dal Bello [38].

Training duration and intensity of both the homebased training programme and the supervised group training schedules will be determined weekly and checked by the therapist by reading out the heart rate monitors, assessing the Borg RPE scale scores and checking the log books. If necessary, individually tailored adjustments to the training schedules will be made by the therapist.

\section{Cognitive Behavioural Therapy (CBT)}

Assuming that living with ALS constitutes stress, we based our CBT approach on a stress-coping model originally proposed by Lazarus \& Folkman (figure 2) [39]. The impact of the symptoms of ALS and the lack of a curative treatment require adaptive mechanisms;

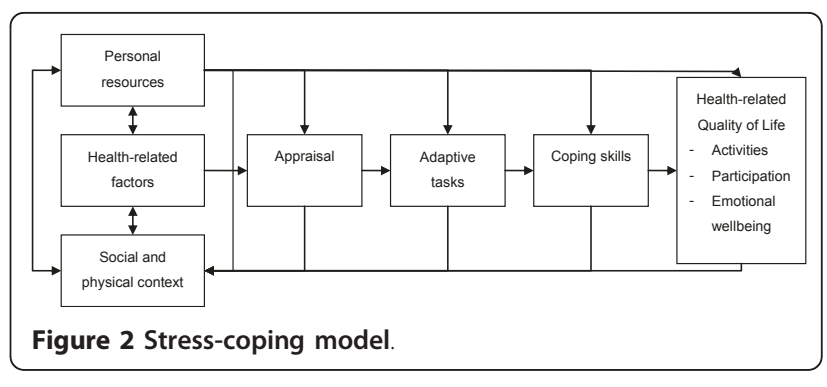

patients have to adjust their internal needs to new external demands. The cognitive process by which this event is interpreted and evaluated is appraisal. Appraisal leads to adaptive tasks, which require coping skills. The required coping skills include a wide range of strategies aimed at stress reduction. Coping influences healthrelated outcomes such as activities and participation, and improves QoL.

The CBT intervention in the FACTS-2-ALS trial will aim to reduce stress by training effective coping skills, modifying dysfunctional cognitions, providing the right amount of disease- and support-related information and encouraging patients to seek social support. Therapy will be tailored to each individual patient and their partner, using behavioural and rational-emotive principles and comprising six "modules". These modules have been designed on the basis of interviews with ALS patients and caregivers, and include the six most important areas identified by the patients with ALS and caregivers who were interviewed: (1) coming to terms with the diagnosis of ALS; (2) coping with mood disorders; (3) maintaining autonomy; (4) mobilizing social support; (5) fear of the future; and (6) maintaining activity levels. Modules will be chosen in the first intake session by the therapists, based on patients' and partners' perceived problems. Depending on the problems, sessions will be held individually or together with the partners. Cognitive Behavioural Therapy (CBT) will consist of individual support for patient and partner by trained psychologists with experience in rehabilitation medicine. Depending on the perceived problems, the psychologists will determine, in consultation with the patients, the number of sessions (5 to 10) to be held during the 16 -week period, each with a duration of 1 hour.

\section{Compliance and attrition}

Compliance will be assessed by recording the number of treatment sessions (AET or CBT) attended. For the patients randomized to AET, the total time spent on aerobic exercise on the cycle ergometer and stepboard at home will also be recorded in a log book. Where applicable, participants will be asked to express their motivation for poor compliance or drop-out. 


\section{Outcomes}

Outcome measurements (table 2) will be obtained at entry into the study (T0), immediately after the 4month intervention period (T1), at 3-month follow-up (short-term follow-up; T2), and at 6-month follow-up (long-term follow-up; T3). The primary outcome measure will be QoL, assessed with the ALS Assessment Questionnaire (ALSAQ-40) [40] and Short Form 36 (SF36) [41], and functioning as assessed with the LASA Physical Activity Questionnaire (LAPAQ) [42], the Impact on Participation and Autonomy questionnaire (IPA) [43], and the Sickness Impact Profile 68 (SIP 68) [44]. Secondary outcome measures will be categorized in accordance with the International Classification of Functioning (ICF) [12] for the domains of body functions and environmental and personal factors. Outcome measures for the domain of body functions (disease severity, survival, cardio-respiratory fitness, functional capacity, muscle strength, lung function, fatigue, sleep disturbances, pain, blood pressure, and weight/height) and will be determined at the three University Medical Centres by a trained research assistant blinded for treatment allocation.

Participants will be asked to complete the questionnaires at home. If they are unable to complete the questionnaires themselves, e.g. due to limited hand function, they will be able to get help from the research assistant. The ALS Functional Rating Scale Revised (ALSFRS-R) [45], Hospital Anxiety and Depression Scale (HADS) [32], and Caregiver Strain Index (CSI) [46] (partner) will be administered by a rehabilitation physician at $\mathrm{T} 0$ and by a research assistant at T1, T2, and T3.

\section{Adverse events}

An adverse event is defined as any undesirable experience or outcome. All adverse events reported spontaneously by the participants or observed by the therapists will be recorded. The adverse events will be followed until they have abated, or until a stable situation has been reached.

\section{Statistical Analyses}

Generalized estimated equations analysis will be used to investigate differences in the effects on primary and secondary outcome measures between the two intervention groups and the usual care group and to investigate the influence of possible effect modifiers. Data will be analyzed according the intention-to-treat principle. Missing data will be imputed by carrying the last observation forward.

\section{Power}

The sample size is based on power analysis, calculated on the basis of a change in the primary outcome during the period from the baseline to 3 months post intervention.
Hardly any useful data for an effective power calculation is available. A recent Cochrane review [7] showed that only two previous trials have been published $[38,47]$ both describing results of a strength training programme, whereas our study will use aerobic exercise oriented training. The primary outcome of both studies was the ALSFRS. Drory [47] found a significant difference between the intervention and control groups of 6.7 points, with a mean SD of 6.7 (Effect Size (ES) 1.0). Dal Bello [38] found a difference of 2.4 with a mean SD of 2.4 (ES 0.6). The ALSFRS is a relevant outcome measure of AET, but less so for the CBT in our trial, and is therefore only a secondary outcome measure in our study. Our primary outcome measure is ALSAQ-40, a measure of QoL which is specifically designed for people with ALS. Based on the ESs of 1.0 and 0.6 found on the ALS-FRS, we expect a difference of 0.8 SD (Alpha 0.05; Beta 0.8), resulting in a minimum required number of 26 participants in each group. Allowing for a $35 \%$ drop-out rate, we aim to include 40 persons per group, so 120 people in total.

\section{Discussion}

The FACTS-2-ALS study will evaluate the effects of $\mathrm{AET}$ and $\mathrm{CTB}$ on improving or preserving functioning and QoL in patients with ALS, compared to usual care alone. The study is characterized by some important strengths.

First, the lack of substantial evidence regarding the role of AET and CBT in people with ALS supports the need to develop further high-quality, sufficiently powered trials. The FACTS-2-ALS study design is a multicentre, single-blinded, randomized controlled clinical trial with long-term follow-up. Patients will be followed up until 6 months after the intervention, which will not only provide information about the maintenance of the effects, but also about any long-term adverse events. We have deliberately chosen a biphasic randomization model with postponed information. This design will prevent patients being disappointed if they are randomized to the control group. Besides, if patients in the control group were fully informed, they might become motivated to seek a similar intervention. Moreover, disappointment may affect the way patients answer the questionnaires about their health and QoL, and consequently influence the study outcomes. A postponed information model can also be considered patientfriendly, as it provides all relevant information stripped of the theoretical and practical complexities of the study design. Moreover, there is no risk of damage caused by not informing the participants, who will be clearly informed at a later stage, with an explanation of the reason why this method was chosen.

Secondly, our design incorporates the recommendations made by Dal Bello [7] regarding a protocol design, 
Table 2 Outcome measures and instrumentation

\begin{tabular}{|c|c|c|c|c|c|}
\hline Primary outcome measures & Instrumentation & TO & T1 & $\mathrm{T} 2$ & T3 \\
\hline \multirow[t]{2}{*}{ Quality of life } & ALS Assessment Questionnaire (ALSAQ-40) [40] & $x$ & $x$ & $x$ & $x$ \\
\hline & Short Form 36 (SF-36) [41] & $x$ & $x$ & $x$ & $x$ \\
\hline \multicolumn{6}{|l|}{ ICF: Activities / participation } \\
\hline Self-reported physical activity & LASA Physical Activity Questionnaire (LAPAQ) [42] & X & $x$ & $x$ & $x$ \\
\hline $\begin{array}{l}\text { Limitations in participation and } \\
\text { autonomy }\end{array}$ & The Impact on Participation and Autonomy questionnaire (IPA) [48] & $x$ & $x$ & $x$ & $x$ \\
\hline Self-perceived functional status & $\begin{array}{l}\text { Sickness Impact Profile } 68 \text { (SIP 68; domains: mobility control, mobility range, social } \\
\text { behaviour) [44] }\end{array}$ & $x$ & $x$ & $x$ & $x$ \\
\hline \multicolumn{6}{|l|}{ Secondary outcome measures } \\
\hline \multicolumn{6}{|l|}{ ICF: Body functions } \\
\hline ALS disease severity & ALS Functional Rating Scale Revised (ALSFRS-R) [45] & $x$ & $x$ & $x$ & $x$ \\
\hline Survival & Survival in months post diagnosis & $x$ & $x$ & $x$ & $x$ \\
\hline Cardio-respiratory fitness & Submaximal exercise test with cycle ergometer [49] & $x$ & $x$ & $x$ & $x$ \\
\hline \multirow[t]{2}{*}{ Functional capacity } & Stairs test [50] & $x$ & $x$ & $x$ & $x$ \\
\hline & Timed Up and Go (TUG) test [51] & $x$ & $x$ & $x$ & $x$ \\
\hline \multirow[t]{2}{*}{ Muscle strength } & MicroFET handheld dynamometer & $x$ & $x$ & $x$ & $x$ \\
\hline & Jamar handheld dynamometer & $x$ & $x$ & $x$ & $x$ \\
\hline \multirow[t]{2}{*}{ Lung function } & Spirometry: forced vital capacity (FVC) [52] & $x$ & $x$ & $x$ & $x$ \\
\hline & Sniff nasal inspiratory pressure (SNIP) [53] & $x$ & $x$ & $x$ & $x$ \\
\hline Fatigue & Checklist Individual Strength (CIS; domain: fatigue) [54] & $x$ & $x$ & $x$ & $x$ \\
\hline Sleep disturbances & Nottingham Health Profile (NHP; sleep dimension) [55] & $x$ & $x$ & $x$ & $x$ \\
\hline Pain & Visual analogue scale, pain (VAS-pain) & $x$ & $x$ & $x$ & $x$ \\
\hline \multirow[t]{4}{*}{ Co-morbidity } & Cumulative IIlness Rating Scale (CIRS) [56] & $x$ & & & \\
\hline & Blood pressure & $x$ & $x$ & $x$ & $x$ \\
\hline & Height / Weight & $x$ & $x$ & $x$ & $x$ \\
\hline & Skinfold & $x$ & $x$ & $x$ & $x$ \\
\hline \multicolumn{6}{|l|}{ ICF: Personal Factors } \\
\hline IIIness cognitions & Ziekte Cognitie Lijst (ZCL) [57] & $x$ & $x$ & $x$ & $x$ \\
\hline Coping & Coping Inventory for Stressful Situations (CISS-21) [58] & $x$ & $x$ & $x$ & $x$ \\
\hline General self-efficacy & General Self-Efficacy Scale (ALCOS-16) [59] & $x$ & & & \\
\hline Personality & Eysenck Personality Questionnaire (EPQ-RSS) [60] & $x$ & & & \\
\hline \multirow[t]{2}{*}{ Mood } & Hospital Anxiety and Depression Scale (HADS) [32] & $x$ & $x$ & $x$ & $x$ \\
\hline & Profile of Mood States (POMS) [61] & $x$ & $x$ & $x$ & $x$ \\
\hline Impact of events & Schokverwerkingslijst (SVL) [62] & $x$ & $x$ & $x$ & $x$ \\
\hline \multicolumn{6}{|l|}{ ICF: Environmental factors } \\
\hline Social support & Social Support List - Discrepancies (SSL-D-12) [63] & $x$ & $x$ & $x$ & $x$ \\
\hline Quality of life (partner) & Short Form 36 (SF-36) [41] & $x$ & $x$ & $x$ & $x$ \\
\hline Coping (partner) & Coping Inventory for Stressful Situations (CISS-21) [58] & $x$ & $x$ & $x$ & $x$ \\
\hline \multirow[t]{2}{*}{ Mood (partner) } & Hospital Anxiety and Depression Scale (HADS) [32] & $x$ & $x$ & $x$ & $x$ \\
\hline & Profile of Mood States (POMS) [61] & $x$ & $x$ & $x$ & $x$ \\
\hline Caregiver strain (partner) & Caregiver Strain Index (CSI) [46] & $x$ & $x$ & $x$ & $x$ \\
\hline Other factors & & T0 & $\mathrm{T} 1$ & $\mathrm{~T} 2$ & $\mathrm{~T} 3$ \\
\hline \multicolumn{2}{|c|}{$\begin{array}{l}\text { Demographic variables (age, gender, ethnicity, marital status, children, education, work, religion, participation in other study) of } \\
\text { patient and partner }\end{array}$} & $x$ & & & \\
\hline
\end{tabular}

T1; pre-treatment, T2; post-treatment, T3; short-term follow-up, T4; long-term follow-up. 
and include: (1) description of the population in terms of specific diagnostic criteria, range of disease severity, and severity of impairments and function to allow readers of the reports to assess the generalizability of the results to their patients or patient groups; (2) effective allocation concealment; (3) blinding of the outcome assessor; (4) a clear description of the exercise intervention, including the mode of exercise, intensity, progression, frequency, duration of each exercise session, duration of the entire programme, muscle groups exercised, supervision of exercise protocol, and compliance assessment; (5) level of activity at baseline; (6) monitoring of adverse events and reporting of motivations for dropout; and (7) standardized outcome measures, i.e. using well-validated outcome measures that are able to assess positive and negative effects of exercise, including measures of muscle function or aerobic capacity, functioning, fatigue, and QoL.

Thirdly, although CBT has proved to be effective in patients with chronic diseases, there are no studies confirming the effects of this approach in patients with ALS. This will be the first study to evaluate the effects of CBT and AET on functioning and QoL in patients with ALS. The treatment protocol was developed by psychologists who have experience with patients with chronic diseases in rehabilitation practice, and was customized to the specific problems in ALS.

However, the study also has some limitations. First, we expect that the inclusion of patients may be difficult, as the disease is very debilitating. Should the intended sample size prove difficult to reach, we will invite more centres to participate in the study, in order to include more patients. Secondly, some of the instruments we will use have not been tested on the population of patients with ALS. In view of the lack of evidence, we will use instruments that have been tested on patient groups with other chronic diseases. Finally, although the 6-month follow-up is a strong point of this study, the relatively long follow-up could cause a high drop-out rate, in view of the devastating nature of the disease. During the study, motivations for drop-out will therefore be closely monitored.

In conclusion, the FACTS-2-ALS study may provide further evidence that may be useful for the multidisciplinary care management of persons with ALS. Results might lead to adaptations of ALS treatment protocols, in order to improve or preserve QoL and functioning in patients with ALS.

\section{List of abbreviations}

ALS: amyotrophic lateral sclerosis; AET: aerobic exercise therapy; CBT: cognitive behavioural therapy; QoL: quality of life; RCT: randomized controlled trial; FACTS-2-ALS: acronym for Fitness And Cognitive behavioural TherapieS/for Fatigue and ACTivitieS in ALS; PPS: Postpoliomyelitis
Syndrome; FSHD: facioscapulohumeral dystrophy; FALS: familial ALS; SALS: Sporadic ALS; NMD: neuromuscular diseases; MD: myotonic dystrophy; RPE: rated perceived exertion; HRR: heart rate reserve; ICF: International

Classification of Functioning, Disability and Health; VO2max: maximal oxygen consumption; AMC: Academic Medical Centre Amsterdam; UMCU: University Medical Centre Utrecht; UMCN: University Medical Centre Nijmegen.

\section{Acknowledgements and Funding}

This study is funded by the Prinses Beatrix Fonds (BPF) (The Dutch Public Fund for Neuromuscular Disorders), The Netherlands Organization for Health Research and Development (ID: ZonMW 89000003).

\section{Author details}

${ }^{1}$ Rudolf Magnus Institute of Neuroscience and Centre of Excellence for Rehabilitation Medicine, University Medical Centre Utrecht and Rehabilitation Centre De Hoogstraat, the Netherlands. ${ }^{2}$ Swiss Paraplegic Research, Nottwil, Switzerland. ${ }^{3}$ Het Roessingh Rehabilitation Centre, Enschede, The Netherlands. ${ }^{4}$ Department of Rehabilitation Medicine, Academic Medical Centre, Amsterdam, the Netherlands. ${ }^{5}$ Radboud University Medical Centre, Nijmegen, the Netherlands.

\section{Authors' contributions}

AvG is the primary investigator and responsible for data collection, analysis and interpretation; she also wrote the manuscript. IvdP, MP, CS, HG, EK, $H v d L, R v V, M v d W, L v d B$ and $E L$ have designed and are supervising the study. All authors have read and approved the manuscript.

\section{Competing interests}

The authors declare that they have no competing interests.

Received: 3 May 2011 Accepted: 14 June 2011 Published: 14 June 2011

\section{References}

1. Pasinelli $\mathrm{P}$, Brown $\mathrm{RH}$ : Molecular biology of amyotrophic lateral sclerosis: insights from genetics. Nat Rev Neurosci 2006, 7:710-723.

2. Brooks BR, Miller RG, Swash M, Munsat TL: El Escorial revisited: revised criteria for the diagnosis of amyotrophic lateral sclerosis. Amyotroph Lateral Scler Other Motor Neuron Disord 2000, 1:293-299.

3. Logroscino G, Traynor BJ, Hardiman O, Chio' A, Couratier P, Mitchell JD, Swingler RJ, Beghi E: Descriptive epidemiology of amyotrophic lateral sclerosis: new evidence and unsolved issues. J Neurol Neurosurg Psychiatry 2008, 79:6-11.

4. Shaw PJ: Molecular and cellular pathways of neurodegeneration in motor neurone disease. J Neurol Neurosurg Psychiatry 2005, 76:1046-1057.

5. Dion PA, Daoud H, Rouleau GA: Genetics of motor neuron disorders: new insights into pathogenic mechanisms. Nat Rev Genet 2009, 10:769-782.

6. Donkervoort S, Siddique T: Amyotrophic Lateral Sclerosis Overview. 1993.

7. Dal Bello-Haas V, Florence JM, Krivickas LS: Therapeutic exercise for people with amyotrophic lateral sclerosis or motor neuron disease. Cochrane Database Syst Rev 2008, CD005229.

8. Giordana MT, Ferrero P, Grifoni S, Pellerino A, Naldi A, Montuschi A Dementia and cognitive impairment in amyotrophic lateral sclerosis: a review. Neurol Sci 2011, 32:9-16.

9. Trail M, Nelson N, Van JN, Appel SH, Lai EC: Major stressors facing patients with amyotrophic lateral sclerosis (ALS): a survey to identify their concerns and to compare with those of their caregivers. Amyotroph Lateral Scler Other Motor Neuron Disord 2004, 5:40-45.

10. Averill AJ, Kasarskis EJ, Segerstrom SC: Psychological health in patients with amyotrophic lateral sclerosis. Amyotroph Lateral Scler 2007, 8:243-254

11. Plahuta JM, McCulloch BJ, Kasarskis EJ, Ross MA, Walter RA, McDonald ER: Amyotrophic lateral sclerosis and hopelessness: psychosocial factors. Soc Sci Med 2002, 55:2131-2140.

12. WHO: International Classification of Functioning Disability and Health (ICF). Geneva; 2001.

13. Engel GL: The need for a new medical model: a challenge for biomedicine. Science 1977, 196:129-136.

14. Bennett RL, Knowlton GC: Overwork weakness in partially denervated skeletal muscle. Clin Orthop 1958, 12:22-29.

15. Coble NO, Maloney FP: Interdisciplinary rehabilitation of multiple sclerosis and neuromuscular disorders. In Effects of exercise in neuromuscular 
disease. Edited by: Maloneym FP, Burks JS, Ringel SP. New York: JB Lippincott; 1985:228-238.

16. Johnson EW, Braddom R: Over-work weakness in facioscapulohumeral muscular dystrophy. Arch Phys Med Rehabil 1971, 52:333-336.

17. Strickland D, Smith SA, Dolliff G, Goldman L, Roelofs Rl: Physical activity, trauma, and ALS: a case-control study. Acta Neurol Scand 1996, 94:45-50

18. Voet NB, van der Kooi EL, Riphagen II, Lindeman E, van Engelen BG, Geurts $\mathrm{ACH}$ : Strength training and aerobic exercise training for muscle disease. Cochrane Database Syst Rev 2010, CD003907.

19. Cup EH, Pieterse AJ, Ten Broek-Pastoor JM, Munneke M, van Engelen BG, Hendricks HT, van der Wilt GJ, Oostendorp RA: Exercise therapy and other types of physical therapy for patients with neuromuscular diseases: a systematic review. Arch Phys Med Rehabil 2007, 88:1452-1464.

20. Kubler A, Winter S, Ludolph AC, Hautzinger M, Birbaumer N: Severity of depressive symptoms and quality of life in patients with amyotrophic lateral sclerosis. Neurorehabil Neural Repair 2005, 19:182-193.

21. Matuz T, Birbaumer N, Hautzinger M, Kubler A: Coping with amyotrophic lateral sclerosis: an integrative view. J Neurol Neurosurg Psychiatry 2010, 81:893-898.

22. Vanden Berg JP, Zandvoort van MJ, Lindeman E, Wokke JHJ, Kalmijn S, Berg van den LHV: Coping styles influence Quality of Life in patients with Amyotrophic Lateral Sclerosis. Submitted 2005.

23. Johnston M, Earll L, Giles M, McClenahan M, Morrison V, Stevens D: Mood as a predictor of disability and survival in patients newly diagnosed with ALS/MND. British Journal of Health Psychology 1999, 4:127-136.

24. Mohr DC, Likosky W, Bertagnolli A, Goodkin DE, Van Der Wende J, Dwyer P, Dick LP: Telephone-administered cognitive-behavioral therapy for the treatment of depressive symptoms in multiple sclerosis. J Consult Clin Psychol 2000, 68:356-361

25. Chambers D, Bagnall AM, Hempel S, Forbes C: Interventions for the treatment, management and rehabilitation of patients with chronic fatigue syndrome/myalgic encephalomyelitis: an updated systematic review. J R Soc Med 2006, 99:506-520.

26. Prins JB, Bleijenberg G, Bazelmans E, Elving LD, de Boo TM, Severens JL, van der Wilt GJ, Spinhoven P, van der Meer JW: Cognitive behaviour therapy for chronic fatigue syndrome: a multicentre randomised controlled trial. Lancet 2001, 357:841-847.

27. Gielissen MFM, Verhagen S, Witjes F, Bleijenberg G: Effects of cognitive behavior therapy in severely fatigued disease-free cancer patients compared with patients waiting for cognitive behavior therapy: a randomized controlled trial. J Clin Oncol 2006, 24:4882-4887.

28. Boter H, van Delden JJM, de Haan RJ, Rinkel GJE: [A modified informedconsent procedure in which the complete information is given retrospectively: no objection from participating patients]. Ned Tijdschr Geneeskd 2005, 149:29-32.

29. Koopman FS, Beelen A, Gerrits KH, Bleijenberg G, Abma TA, de Visser M, Nollet F: Exercise therapy and cognitive behavioural therapy to improve fatigue, daily activity performance and quality of life in postpoliomyelitis syndrome: the protocol of the FACTS-2-PPS trial. BMC Neurol 2010, 10:8.

30. Voet NBM, Bleijenberg G, Padberg GW, van Engelen BGM, Geurts ACH: Effect of aerobic exercise training and cognitive behavioural therapy on reduction of chronic fatigue in patients with facioscapulohumeral dystrophy: protocol of the FACTS-2-FSHD trial. BMC Neurol 2010, 10:56.

31. Abma TA: The Practice and Politics of Responsive Evaluation. American Journal of Evaluation 2006, 27:31-43.

32. Zigmond AS, Snaith RP: The hospital anxiety and depression scale. Acta Psychiatr Scand 1983, 67:361-370.

33. Scott NW, McPherson GC, Ramsay CR, Campbell MK: The method of minimization for allocation to clinical trials. a review. Control Clin Trials 2002, 23:662-674.

34. Vanden Berg JP, Kalmijn S, Lindeman E, Veldink JH, de VM, Van der Graaff MM, Wokke JH, van den Berg LH: Multidisciplinary ALS care improves quality of life in patients with ALS. Neurology 2005, 65:1264-1267.

35. Borg GA: Psychophysical bases of perceived exertion. Med Sci Sports Exerc 1982, 14:377-381.

36. American College of Sports Medicine Position Stand: The recommended quantity and quality of exercise for developing and maintaining cardiorespiratory and muscular fitness, and flexibility in healthy adults. Med Sci Sports Exerc 1998, 30:975-991.
37. Durstine JL, Moore GE: ACSM's Exercise Management for Persons with Chronic Disease and Disabilities. Human Kinetics Champaign; 2003.

38. Dal Bello-Haas VD, Florence JM, Kloos AD, Scheirbecker J, Lopate G, Hayes SM, Pioro EP, Mitsumoto H: A randomized controlled trial of resistance exercise in individuals with ALS. Neurology 2007, 68:2003-2007.

39. Lazarus RS, Folkman S: Stress, appraisal and coping. New York: Springer; 1984

40. Jenkinson C, Fitzpatrick R, Brennan C, Swash M: Evidence for the validity and reliability of the ALS assessment questionnaire: the ALSAQ-40. Amyotroph Lateral Scler Other Motor Neuron Disord 1999, 1:33-40.

41. Ware JE, Snow KK, Kosinski M, Gandek B: SF-36 health survey manual and interpretation guide. Boston (MA): The Health Institute, New England Medical Center; 1993.

42. Stel VS, Smit JH, Pluijm SMF, Visser M, Deeg DJH, Lips P: Comparison of the LASA Physical Activity Questionnaire with a 7-day diary and pedometer. J Clin Epidemiol 2004, 57:252-258.

43. Cardol M, de Haan RJ, van den Bos GA, de Jong BA, de Groot IJ: The development of a handicap assessment questionnaire: the Impact on Participation and Autonomy (IPA). Clin Rehabil 1999, 13:411-419.

44. Bergner M, Bobbitt RA, Carter WB, Gilson BS: The Sickness Impact Profile: development and final revision of a health status measure. Med Care 1981, 19:787-805

45. Cedarbaum JM, Stambler N, Malta E, Fuller C, Hilt D, Thurmond B, Nakanishi A: The ALSFRS-R: a revised ALS functional rating scale that incorporates assessments of respiratory function. BDNF ALS Study Group (Phase III). J Neurol Sci 1999, 169:13-21.

46. Post MWM, Festen $H$, van de Port IG, Visser-Meily JMA: Reproducibility of the Caregiver Strain Index and the Caregiver Reaction Assessment in partners of stroke patients living in the Dutch community. Clin Rehabil 2007, 21:1050-1055.

47. Drory VE, Goltsman E, Reznik JG, Mosek A, Korczyn AD: The value of muscle exercise in patients with amyotrophic lateral sclerosis. J Neurol Sci 2001, 191:133-137.

48. Cardol M, de Haan RJ, de Jong BA, van den Bos GA, de Groot IJ: Psychometric properties of the Impact on Participation and Autonomy Questionnaire. Arch Phys Med Rehabil 2001, 82:210-216.

49. Astrand PO, Ryhming Gl: A nomogram for calculation of aerobic capacity (physical fitness) from pulse rate during sub-maximal work. J Appl Physiol 1954, 7:218-221.

50. Lindeman E, Leffers P, Spaans F, Drukker J, Reulen J, Kerckhoffs M, Koke A: Strength training in patients with myotonic dystrophy and hereditary motor and sensory neuropathy: a randomized clinical trial. Arch Phys Med Rehabil 1995, 76:612-620.

51. Podsiadlo D, Richardson S: The timed "Up \& Go": a test of basic functional mobility for frail elderly persons. J Am Geriatr Soc 1991, 39:142-148.

52. Leigh PN, Swash M, Iwasaki $Y$, Ludolph A, Meininger $V$, Miller RG, Mitsumoto H, Shaw P, Tashiro K, Van Den Berg L: Amyotrophic latera sclerosis: a consensus viewpoint on designing and implementing a clinical trial. Amyotroph Lateral Scler Other Motor Neuron Disord 2004, 5:84-98

53. Fitting JW, Paillex R, Hirt L, Aebischer P, Schluep M: Sniff nasal pressure: a sensitive respiratory test to assess progression of amyotrophic lateral sclerosis. Ann Neurol 1999, 46:887-893.

54. Vercoulen JH, Swanink CM, Fennis JF, Galama JM, van der Meer JW, Bleijenberg G: Dimensional assessment of chronic fatigue syndrome. $J$ Psychosom Res 1994, 38:383-392.

55. Hunt SM, McEwen J, McKenna SP: Measuring health status: a new tool for clinicians and epidemiologists. J R Coll Gen Pract 1985, 35:185-188.

56. Linn BS, Linn MW, Gurel L: Cumulative illness rating scale. J Am Geriatr Soc 1968, 16:622-626.

57. Evers AW, Kraaimaat FW, van Lankveld W, Jongen PJ, Jacobs JW, Bijlsma JW: Beyond unfavorable thinking: the illness cognition questionnaire for chronic diseases. J Consult Clin Psychol 2001, 69:1026-1036.

58. Endler NS, Parker JDA: Coping Inventory for Stressful Situations (CISS) Manual. Multi-Health Systems Toronto, Canada; 1999.

59. Bosscher RJ, Smit JH: Confirmatory factor analysis of the General SelfEfficacy Scale. Behav Res Ther 1998, 36:339-343.

60. Eysenck HJ, Eysenck SBG: Manual of the Eysenck Personality Questionnaire. London: Hodder and Stoughton; 1975. 
61. Shacham S: A shortened version of the Profile of Mood States. J Pers Assess 1983, 47:305-306.

62. Horowitz M, Wilner N, Alvarez W: Impact of Event Scale: a measure of subjective stress. Psychosom Med 1979, 41:209-218.

63. Van Sonderen E: Sociale Steun Lijst-Interacties (SSL-I) en Sociale Steun Lijst -Discrepanties (SSL-D). Noordelijk Centrum voor

Gezondheidsvraagstukken Groningen; 1993.

\section{Pre-publication history}

The pre-publication history for this paper can be accessed here: http://www.biomedcentral.com/1471-2377/11/70/prepub

doi:10.1186/1471-2377-11-70

Cite this article as: van Groenestijn et al.: Effects of aerobic exercise

therapy and cognitive behavioural therapy on functioning and quality of life in amyotrophic lateral sclerosis: protocol of the FACTS-2-ALS trial. BMC Neurology 2011 11:70.

\section{Submit your next manuscript to BioMed Central} and take full advantage of:

- Convenient online submission

- Thorough peer review

- No space constraints or color figure charges

- Immediate publication on acceptance

- Inclusion in PubMed, CAS, Scopus and Google Scholar

- Research which is freely available for redistribution

Submit your manuscript at www.biomedcentral.com/submit 